\title{
Calderon Multiplicative Preconditioner for the PMCHWT Equation Applied to Chiral Media
}

\author{
Yves Beghein \\ Department of Information Technology \\ Ghent University \\ 9000 Gent, Belgium
}

Daniël De Zutter

Department of Information Technology

Ghent University

9000 Gent, Belgium

\author{
Kristof Cools \\ Department of Information Technology \\ Ghent University \\ 9000 Gent, Belgium
}

\author{
Francesco P. Andriulli \\ Microwave Department \\ TELECOM Bretagne \\ 29238 Brest Cedex 3 , France
}

\author{
Eric Michielssen \\ Dept. of Electrical Engineering and Computer Science \\ University of Michigan \\ Ann Arbor, Michigan 48109, USA
}

\begin{abstract}
In this contribution, a Calderón preconditioned algorithm for the modeling of scattering of time harmonic electromagnetic waves by a chiral body is introduced. The construction of the PMCHWT in the presence of chiral media is revisited. Since this equation reduces to the classic PMCHWT equation when the chirality parameter tends to zero, it shares its spectral properties. More in particular, it suffers from dense grid breakdown. Based on the work in [1], [2], a regularized version of the PMCHWT equation is introduced. A discretization scheme is described. Finally, the validity and spectral properties are studied numerically. More in particular, it is proven that linear systems arising in the novel scheme can be solved in a small number of iterations, regardless the mesh parameter.
\end{abstract}

\section{INTRODUCTION}

The scattering of time-harmonic electromagnetic fields by perfectly conducting and penetrable media can be modeled by boundary integral equations. In the so-called direct method, these equations are constructed from the Maxwell equations by leveraging the Stratton-Chu representation formulas [3]. These representation formulas represent the electromagnetic field within a homogeneous body as integral expressions involving the tangential components of the traces of the electromagnetic field inside this body. As a special case, the value of the tangential components of the traces of the electromagnetic field can be represented, thus yielding equations for these traces. By combining said representations in the different domains, different equations that can be solved uniquely for the traces of the electromagnetic field can be constructed. The two most widespread equations are the Poggio-Miller-ChangHarrington-Wu-Tsai equation and the Müller (PMCHWT) equation.

In the high conductivity limit, the PMCHWT equation reduces to the electric field integral equation (EFIE) used to model scattering by a perfectly conducting body, while the Müller equation reduces to the magnetic field integral equation (MFIE). Therefore, it is not surprising that the spectral properties of the PMCHWT and Müller equations resemble those of the EFIE and MFIE, respectively. Indeed, like the spectrum of the EFIE, the spectrum of the PMCHWT equation comprises two branches, one accumulating at zero and one accumulating at infinity. The Müller equation, in contrast, is a second kind equation, and therefore its spectrum accumulates at a finite non zero value.

The spectral properties of these continuous equations are reflected in the eigenvalue distribution of the system matrices resulting upon discretization. The system matrices resulting from discretizations of the PMCHWT equation possess an eigenvalue spectrum comprising to subsets. One subset stretches along the positive imaginary axis and accumulates at zero, while the other subset stretches along the negative imaginary axis and accumulates at infinity. The systems are thus ill-conditioned, and moreover strongly indefinite. This renders the solution by iterative algorithms hard to impossible. The system matrices resulting from discretization of the Müller equation posses an eigenvalue spectrum comprising only one set accumulating at a positive real value. The system matrices are thus both well-conditioned and up to a compact perturbation positive definite. This renders the solution by iterative algorithms easy. Lamentably, solutions to equations of the classically discretized Müller equation are less accurate than those of the classically discretized PMCHWT equation.

One possibility is to seek for more intricate discretization schemes for the Müller/MFIE equation such that the resulting linear systems yield more accurate solutions. This possibility has been investigated in [4], with success. Another possibility is the preconditioning of the linear system resulting upon discretization of the PMCHWT/EFIE equation such that the system matrix of the preconditioned system possesses an eigenvalue spectrum with beneficial distribution in the complex plane. This method has been investigated in [2] and hinges on the self-regularizing property of the operator involved. The work in [2] is an extension of that in [1] where the PEC case was studied to the case of penetrable bodies.

In this contribution, the methods developed in [1], [2] will be extended to the case of scattering by chiral media. It will be clear from the exposition that this extension is non trivial because the representation formulas involved in the 
construction of the boundary integral equations contain more contributions.

First, the construction of the aforementioned boundary integral representations is revisited. From these, the equivalent of the PMCHWT equation is constructed. Next the discretization scheme is discussed. The resulting algorithm to model scattering by chiral bodies will be validated by comparison to the Mie series. It will be shown numerically that the condition number grows arbitrarily when the mesh parameter decreases. Finally, a Calderón multiplicative preconditioner will be introduced. It will be shown that the preconditioned linear system is wellconditioned and that it can be solved efficienty by iterative algorithms.

\section{EQUATIONS}

Consider a scatterer with surface $\Gamma$ and outward pointing normal $\hat{\boldsymbol{n}}$. The scatterer is illuminated by a time harmonic electromagnetic field $\left(\boldsymbol{e}^{i n c}, \boldsymbol{h}^{i n c}\right)$ varying with angular frequency $\omega$. The medium surrounding the scatterer is characterized by a permittivity $\epsilon_{0}$ and a permeability $\mu_{0}$. Let $\eta_{0}=\sqrt{\mu_{0} / \epsilon_{0}}$ and $k_{0}=\omega \sqrt{\mu_{0} \epsilon_{0}}$ be the corresponding impedance and wave number, respectively. The electromagnetic fields outside the scatterer are solutions of the StrattonChew representation formulas for the tangential components of the traces of the electric and magnetic fields:

$$
\begin{aligned}
& \left(\begin{array}{c}
\tilde{\boldsymbol{e}}_{0} \times \hat{\boldsymbol{n}} \\
\hat{\boldsymbol{n}} \times \tilde{\boldsymbol{h}}_{0}
\end{array}\right)=\left(\begin{array}{c}
\tilde{\boldsymbol{e}}^{i n c} \times \hat{\boldsymbol{n}} \\
\hat{\boldsymbol{n}} \times \tilde{\boldsymbol{h}}^{i n c}
\end{array}\right) \\
& +\left(\begin{array}{cc}
-K^{0}+\frac{1}{2} & \eta_{0} T^{0} \\
-T^{0} / \eta_{0} & -K^{0}+\frac{1}{2}
\end{array}\right)\left(\begin{array}{c}
\tilde{\boldsymbol{e}}_{0} \times \hat{\boldsymbol{n}} \\
\hat{\boldsymbol{n}} \times \tilde{\boldsymbol{h}}_{0}
\end{array}\right)
\end{aligned}
$$

where

$$
\begin{aligned}
T_{0} \boldsymbol{f}(\boldsymbol{r}) & =\frac{1}{j k_{0}} \int_{\Gamma} \hat{\boldsymbol{n}} \times \nabla \frac{e^{-j k_{0} R}}{4 \pi R} \nabla^{\prime} \cdot \boldsymbol{f}\left(\boldsymbol{r}^{\prime}\right) d \gamma\left(\boldsymbol{r}^{\prime}\right) \\
& =-j k_{0} \int_{\Gamma} \hat{\boldsymbol{n}} \times \frac{e^{-j k_{0} R}}{4 \pi R} \boldsymbol{f}\left(\boldsymbol{r}^{\prime}\right), d \gamma\left(\boldsymbol{r}^{\prime}\right), \\
K_{0} \boldsymbol{f}(\boldsymbol{r}) & =\int_{\Gamma} \hat{\boldsymbol{n}} \times \nabla \frac{e^{-j k_{0} R}}{4 \pi R} \times \boldsymbol{f}\left(\boldsymbol{r}^{\prime}\right) d \gamma\left(\boldsymbol{r}^{\prime}\right) .
\end{aligned}
$$

The scatterer is filled with a chiral medium characterized by a permittivity $\epsilon$, a permeability $\mu$, and a chirality parameter $\kappa$. A number of different models exist to describe chiral materials [5]. For time-harmonic fields, most of these models result in Maxwell equations of the following form:

$$
\begin{aligned}
& \nabla \times \boldsymbol{e}=-j \omega(-j \kappa \sqrt{\epsilon \mu} \boldsymbol{e}+\mu \boldsymbol{h}), \\
& \nabla \times \boldsymbol{h}=j \omega(\epsilon \boldsymbol{e}+j \kappa \sqrt{\epsilon \mu} \boldsymbol{h}) .
\end{aligned}
$$

Although these equations are more involved than the classic Maxwell equations, they can be brought in the same form following the reasoning in described in [6]. First, the Bohren transformation [5] is applied to the fields:

$$
\left(\begin{array}{c}
\tilde{e}_{1} \\
\tilde{e}_{2}
\end{array}\right)=\left(\begin{array}{cc}
1 & -j \eta \\
1 & j \eta
\end{array}\right)\left(\begin{array}{l}
\boldsymbol{e} \\
\boldsymbol{h}
\end{array}\right) \text {. }
$$

Here, $\eta=\sqrt{\mu / \epsilon}$. Note that in general this quantity looses its physical meaning of an impedance. Substitution of the electric and magnetic field in favor of these auxiliary electric fields diagonalizes the Maxwell equations, yielding

$$
\begin{aligned}
& \nabla \times \tilde{\boldsymbol{e}}_{1}=\gamma_{1} \tilde{\boldsymbol{e}}_{1}, \\
& \nabla \times \tilde{\boldsymbol{e}}_{2}=-\gamma_{2} \tilde{\boldsymbol{e}}_{2},
\end{aligned}
$$

with

$$
\begin{aligned}
& \gamma_{1}=\omega \sqrt{\epsilon \mu}(1-\kappa), \\
& \gamma_{2}=\omega \sqrt{\epsilon \mu}(1+\kappa)
\end{aligned}
$$

the propagation coefficient of their respective modes. Although at first inspection is seems that the two equations are completely decoupled, in practice they cannot be solved separately since the complete space is not filled with the same chiral medium, and these contributions couple through the continuity conditions imposed at the boundaries of the medium. Finally, introduce the following auxiliary magnetic fields:

$$
\begin{aligned}
\tilde{\boldsymbol{h}}_{1} & =-\frac{1}{j \omega_{1} \mu} \nabla \times \tilde{\boldsymbol{e}}_{1}, \\
\tilde{\boldsymbol{h}}_{2} & =-\frac{1}{j \omega_{2} \mu} \nabla \times \tilde{\boldsymbol{e}}_{2} .
\end{aligned}
$$

with $\omega_{1}=(1-\kappa) \omega$ and $\omega_{2}=(1+\kappa) \omega$. Taking into account (7-8) and (13-14), the inversion of (6) can written as

$$
\left(\begin{array}{l}
\boldsymbol{e} \\
\boldsymbol{h}
\end{array}\right)=\frac{1}{2}\left(\begin{array}{c}
\tilde{\boldsymbol{e}}_{1} \\
\tilde{\boldsymbol{h}}_{1}
\end{array}\right)+\frac{1}{2}\left(\begin{array}{c}
\tilde{\boldsymbol{e}}_{2} \\
\tilde{\boldsymbol{h}}_{2}
\end{array}\right) .
$$

The auxiliary electric and magnetic field can be seen to fulfill to following set of formal Maxwell equations:

$$
\begin{aligned}
& \nabla \times \tilde{\boldsymbol{e}}_{i}=-j \omega_{i} \tilde{\boldsymbol{h}}_{i}, \\
& \nabla \times \tilde{\boldsymbol{h}}_{i}=j \omega_{i} \tilde{\boldsymbol{e}}_{i},
\end{aligned}
$$

for $i=1,2$. Let $k_{i}=\omega_{i} \sqrt{\epsilon \mu}, i=1,2$ be the effective wave numbers. Since the auxiliary field pairs obey formal Maxwell equations, it can be immediately concluded that they can be represented by the Stratton-Chu representation formulas. In particular, for the tangential components of their traces,

$$
\left(\begin{array}{c}
\tilde{\boldsymbol{e}}_{i} \times \hat{\boldsymbol{n}} \\
\hat{\boldsymbol{n}} \times \tilde{\boldsymbol{h}}_{i}
\end{array}\right)=\left(\begin{array}{cc}
-K^{i}+\frac{1}{2} & \eta T^{i} \\
-T^{i} / \eta & -K^{i}+\frac{1}{2}
\end{array}\right)\left(\begin{array}{c}
\tilde{\boldsymbol{e}}_{i} \times \hat{\boldsymbol{n}} \\
\hat{\boldsymbol{n}} \times \tilde{\boldsymbol{h}}_{i}
\end{array}\right)
$$

for $i=1,2$. The definitions of $\left(T^{i}, K^{i}\right)$ are analogous to those of $\left(T^{0}, K^{0}\right)$. The representation formulas for the original fields can be constructed from these using (6) and (15):

$$
\begin{aligned}
& \left(\begin{array}{c}
\boldsymbol{e} \times \hat{\boldsymbol{n}} \\
\hat{\boldsymbol{n}} \times \boldsymbol{h}
\end{array}\right)= \\
& \left(\begin{array}{cc}
-\left(K^{+}-j T^{-}\right)+\frac{1}{2} & \eta\left(T^{+}+j K^{-}\right) \\
-\left(T^{+}+j K^{-}\right) / \eta & -\left(K^{+}-j T^{-}\right)+\frac{1}{2}
\end{array}\right)\left(\begin{array}{c}
\boldsymbol{e} \times \hat{\boldsymbol{n}} \\
\hat{\boldsymbol{n}} \times \boldsymbol{h}
\end{array}\right)
\end{aligned}
$$

with $K^{ \pm}=\left(K^{1}-K^{2}\right) / 2$ and $T^{ \pm}=\left(T^{1}-T^{2}\right) / 2$.

The PMCHWT equation can be constructed by subtracting the outer and inner representation formulas, giving rise to [6]

$$
-\left(\begin{array}{c}
\boldsymbol{e}^{i n c} \times \hat{\boldsymbol{n}} \\
\hat{\boldsymbol{n}} \times \boldsymbol{h}^{i n c}
\end{array}\right)=P\left(\begin{array}{c}
\boldsymbol{e} \times \hat{\boldsymbol{n}} \\
\hat{\boldsymbol{n}} \times \boldsymbol{h}
\end{array}\right)
$$


with

$P=\left(\begin{array}{cc}K^{0}+\left(K^{+}-j T^{-}\right) & -\eta_{o} T^{0}-\eta\left(T^{+}+j K^{-}\right) \\ T^{0} / \eta_{0}+\left(T^{+}+j K^{-}\right) / \eta & K^{0}+\left(K^{+}-j T^{-}\right)\end{array}\right)$

Notice that the structure of the operator involved is significantly more intricate than in the case of non chiral materials. More in particular, both the single layer potential $T$ and double layer potential $K$ operators appear in every block of the operator. In the numerical example section, it will be shown that linear systems resulting upon discretization of this system are ill-conditioned, creating the need for a regularized equation. Inspired by the results in [2] and [1], the following regularized equation is proposed:

$$
-P\left(\begin{array}{c}
\boldsymbol{e}^{i n c} \times \hat{\boldsymbol{n}} \\
\hat{\boldsymbol{n}} \times \boldsymbol{h}^{i n c}
\end{array}\right)=P^{2}\left(\begin{array}{c}
\boldsymbol{e} \times \hat{\boldsymbol{n}} \\
\hat{\boldsymbol{n}} \times \boldsymbol{h}
\end{array}\right) .
$$

\section{DisCRETIZATION}

Following literature, equation (20) is discretized by approximating $\Gamma$ by a triangular mesh comprising $N$ edges. The largest among the triangle's diameters is dubbed the mesh parameter $h$. Subordinate to this mesh, the Rao-Wilton-Glisson divergence conforming basis functions $\boldsymbol{f}_{n}, n=1, \ldots, N$ are defined. The unknown current densities are approximated by expansions in these basis functions. The expansions are substituted in (20). Finally, (20) is tested by curl conforming Rao-Wilton-Glisson functions $\hat{\boldsymbol{n}} \times \boldsymbol{f}_{n}, N=1, \ldots, N$. The following linear system arises:

$$
\mathbf{P}_{f f} \cdot\left(\begin{array}{c}
\mathrm{M}_{f} \\
\mathrm{~J}_{f}
\end{array}\right)=-\left(\begin{array}{c}
\mathrm{M}_{f}^{i n c} \\
\mathrm{~J}_{f}^{i n c}
\end{array}\right) .
$$

where $\mathbf{P}_{f f}$ is

$$
\left(\begin{array}{cc}
\mathbf{K}_{f f}^{0}+\left(\mathbf{K}_{f f}^{+}-j \mathbf{T}_{f f}^{-}\right) & -\eta_{0} \mathbf{T}_{f f}^{0}-\eta\left(\mathbf{T}_{f f}^{+}+j \mathbf{K}_{f f}^{-}\right) \\
\mathbf{T}_{f f}^{0} / \eta_{0}+\left(\mathbf{T}_{f f}^{+}+j \mathbf{K}_{f f}^{-}\right) / \eta & \mathbf{K}_{f f}^{0}+\left(\mathbf{K}_{f f}^{+}-j \mathbf{T}_{f f}^{-}\right)
\end{array}\right)
$$

Here,

$$
\mathbf{O}_{f f}=\int_{\Gamma} d \gamma(\boldsymbol{r})(\hat{\boldsymbol{n}} \times \boldsymbol{f})(\boldsymbol{r}) \cdot O \boldsymbol{f}(\boldsymbol{r}),
$$

with $O$ any of the operators in (21). Furthermore $\mathrm{M}_{f}$ and $\mathrm{J}_{f}$ contain the expansion coefficients of $\boldsymbol{e} \times \hat{\boldsymbol{n}}$ and $\hat{\boldsymbol{n}} \times \boldsymbol{h}$ with regards to $\boldsymbol{f}_{n}, n=1, \ldots, N$, respectively, and

$$
\begin{aligned}
\left(\mathrm{M}_{f}^{i n c}\right)_{n} & =\int_{\Gamma} d \gamma(\boldsymbol{r})\left(\hat{\times} \boldsymbol{f}_{n}\right) \cdot\left(\boldsymbol{e}^{i n c} \times \hat{\boldsymbol{n}}\right), \\
\left(\mathrm{J}_{f}^{i n c}\right)_{n} & =\int_{\Gamma} d \gamma(\boldsymbol{r})\left(\hat{\times} \boldsymbol{f}_{n}\right) \cdot\left(\hat{\boldsymbol{n}} \times \boldsymbol{h}^{i n c}\right) .
\end{aligned}
$$

To stably discretize (22), Buffa-Christiansen functions [7] $\boldsymbol{g}_{n}$ and $\hat{\boldsymbol{n}} \times \boldsymbol{g}_{n}$ subordinate to the same mesh need to be introduced in addition to the Rao-Wilton-Glisson basis functions. The resulting Calderón preconditioned PMCHWT equation is

$$
\mathbf{P}_{g g} \cdot \mathbf{S}_{f g}^{-1} \cdot \mathbf{P}_{f f} \cdot\left(\begin{array}{c}
\mathrm{M} \\
\mathrm{J}
\end{array}\right)=-\mathbf{P}_{g g} \cdot \mathbf{S}_{f g}^{-1} \cdot\left(\begin{array}{c}
\mathrm{M}^{i n c} \\
\mathrm{~J}^{i n c}
\end{array}\right),
$$

where $\mathbf{S}_{f g}$ is

$$
\left(\begin{array}{cc}
\mathbf{G}_{f g}^{-1} & \mathbf{0} \\
\mathbf{0} & \mathbf{G}_{f g}^{-1}
\end{array}\right)
$$

and $\left(\mathbf{G}_{f g}\right)_{m, n}=\int_{\Gamma} d \gamma(\boldsymbol{r})\left(\hat{\boldsymbol{n}} \times \boldsymbol{f}_{m}(\boldsymbol{r})\right) \cdot \boldsymbol{g}_{n}(\boldsymbol{r})$.

\section{NumericAl Results}

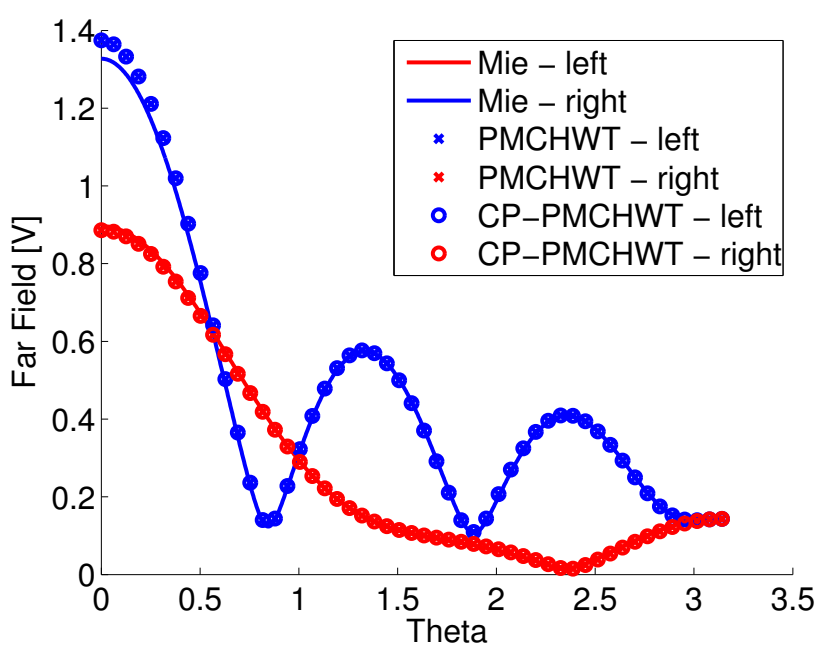

Fig. 1. Comparison of the far field resulting from the PMCHWT and CPPMCHWT solvers with that predicted by the Mie series

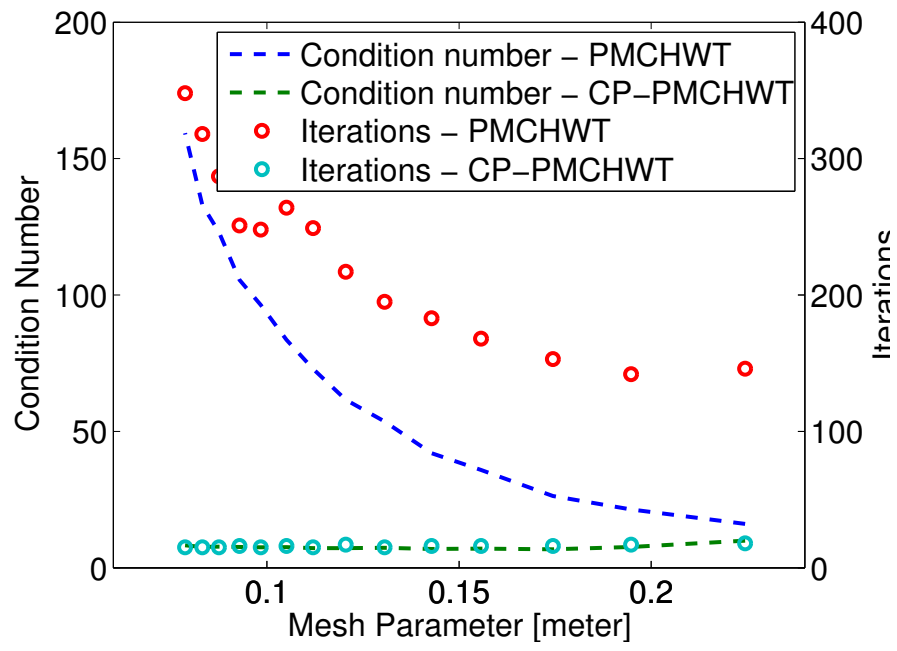

Fig. 2. Condition number and iteration count as a function of the mesh parameter.

In this section, both the classic discretization of the PMCHWT for scattering by chiral media as the discretization of the Calderón preconditioned PMCHWT described above will be used to model scattering by a sphere.

The sphere is illuminated by a plane wave

$$
\boldsymbol{e}^{i n c}=\hat{\boldsymbol{p}} e^{-j \sqrt{\epsilon_{0} \mu_{0}} \omega z}
$$

with $\omega=1.884 \cdot 10^{9}$ per second, and $\hat{\boldsymbol{p}}$ the polarization. Both left handed $(\hat{\boldsymbol{p}}=\hat{\boldsymbol{x}}+j \hat{\boldsymbol{y}})$ and right handed excitations $(\hat{\boldsymbol{p}}=\hat{\boldsymbol{x}}-j \hat{\boldsymbol{y}})$ were used. The sphere is characterized by a permittivity $\epsilon=2 \epsilon_{0}$, a permeability $\mu=\mu_{0}$ and a chirality 
parameter $\kappa=0.5$. The simulation was performed at mesh parameters decreasing from 0.225 meter to 0.075 meter.

First, to validate the method, the far field was compared to the far field predicted by the Mie series. Simulations were performed at a mesh parameter of 0.13 meter. There is a clear influence of chirality (Fig. 1). Results from both polarization and from both the PMCHWT and the Calderón preconditioned PMCHWT solvers agree with the Mie series.

Next, at each mesh parameter, the condition number of the system matrix and the number of iterations needed to reach a relative error of $1 \cdot 10^{-6}$ was recorded. It is clear that while both the condition number and iteration count of the PMCHWT solver increases quadratically, the condition number and iteration count of the CP-PMCHWT solver remains stable (Fig. 2).

\section{REFERENCES}

[1] F.P. Andriulli, K. Cools, H. Bä̈ci, F. Olyslager, A. Buffa, S. Christiansen, and E. Michielssen. A multiplicative Calderon preconditioner for the electric field integral equation. IEEE Transactions on Antennas and Propagation, 56(8):2398-2412, August 2008.

[2] K. Cools, F.P. Andriulli, and F. Olyslager. A Calderón preconditioned PMCHWT equation. In International Conference on Electromagnetics in Advanced Applications, pages 521-524, Torino, September 2009.

[3] R.F. Harrington. Boundary integral formulations for homogeneous material bodies. Journal of Electromagnetic Waves and Applications, 3(1):1$15,1989$.

[4] K. Cools, F.P. Andriulli, F. Olyslager, and E. Michielssen. Improving the mfie's accuracy by using a mixed discretization. In Antennas and Propagation Society International Symposium, pages 1-4, June 2009.

[5] A. Lakhtakia, V.K. Varadan, and V.V. Varadan. Time-Harmonic Electromagnetic Fields in Chiral Media, volume 335 of Lecture Notes in Physics. Springer-Verlag, 1989.

[6] H. Ammari and J.C. Nedelec. Time-harmonic electromagnetic fields in chiral media. Modern Mathematical Methods in Diffraction Theory and its Applications in Engineering, pages 174-202, 1997.

[7] A. Buffa and S. Christiansen. A dual finite element complex on the barycentric refinement. Math. of Comp., 260:1743-1769, 2007. 